\title{
Yagzhev polynomial mappings: on the structure of the Taylor expansion of their local inverse
}

\author{
by Gianluca Gorni (Udine) and Gaetano Zampieri (Messina)
}

Dedicated to Aldo Bressan on the occasion of his 70th birthday

\begin{abstract}
It is well known that the Jacobian conjecture follows if it is proved for the special polynomial mappings $f: \mathbb{C}^{n} \rightarrow \mathbb{C}^{n}$ of the Yagzhev type: $f(x)=x-G(x, x, x)$, where $G$ is a trilinear form and $\operatorname{det} f^{\prime}(x) \equiv 1$. Drużkowski and Rusek [7] showed that if we take the local inverse of $f$ at the origin and expand it into a Taylor series $\sum_{k>1} \Phi_{k}$ of homogeneous terms $\Phi_{k}$ of degree $k$, we find that $\Phi_{2 m+1}$ is a linear combination of certain $m$-fold "nested compositions" of $G$ with itself. If the Jacobian Conjecture were true, $f^{-1}$ should be a polynomial mapping of degree $\leq 3^{n-1}$ and the terms $\Phi_{k}$ ought to vanish identically for $k>3^{n-1}$. We may wonder whether the reason why $\Phi_{2 m+1}$ vanishes is that each of the nested compositions is somehow zero for large $m$. In this note we show that this is not at all the case, using a polynomial mapping found by van den Essen for other purposes.
\end{abstract}

A famous problem on which many mathematicians have worked is the Jacobian conjecture, originated by Keller in [10]: is a polynomial mapping $f: \mathbb{C}^{n} \rightarrow \mathbb{C}^{n}$ with nonzero constant Jacobian determinant necessarily oneto-one? As such the problem is still open, although many interesting results have been obtained in connection with it. One of them is specially nice and easy to state: a polynomial mapping $\mathbb{C}^{n} \rightarrow \mathbb{C}^{n}$ that is one-to-one must always be onto and the inverse is itself a polynomial mapping, that is to say, it is a polynomial automorphism of $\mathbb{C}^{n}$. This fact was proved by Białynicki-Birula and Rosenlicht [2], and alternative proofs can be found in Drużkowski [6] and Rudin [11]. The degree of the inverse (the maximum of the degrees of the components) is also estimated by the $(n-1)$ th power of the degree of $f$ (Bass, Connell and Wright [1]).

One line of approach to the Jacobian conjecture is to look for the Taylor series of the local inverse of $f$ around, say, the origin, which exists and is

1991 Mathematics Subject Classification: Primary 32H99.

We thank the Mathematics Department of the University of Padova, Italy, for logistic support. We are grateful to Ludwik Drużkowski for a critical reading of a draft of the paper. 
analytic in a neighbourhood of $f(0)$ because the Jacobian determinant does not vanish. The terms in the Taylor expansion can in principle be computed from $f$ by repeatedly differentiating the identity $f\left(f_{\text {loc }}^{-1}(x)\right) \equiv x$, for $x$ near the origin of $\mathbb{C}^{n}$. The formulas become of course terribly complicated very quickly, as one can imagine. The amazing thing is that, if the Jacobian conjecture is true, those complicated formulas should vanish identically after a finite number of steps, because $f_{\mathrm{loc}}^{-1}$ must be a polynomial.

However, at least for a special case there used to be a faint hope to find arguments from within the Taylor approach that may account for the vanishing of the high-order terms. This was the case of the polynomial mapping of the special Yagzhev type: maps of the form

$$
f(x)=x+g(x)
$$

where $g: \mathbb{C}^{n} \rightarrow \mathbb{C}^{n}$ is a polynomial mapping homogeneous of degree 3 . It can be verified that the constant Jacobian condition is equivalent to the Jacobian matrix $g^{\prime}(x)$ being nilpotent: $g^{\prime}(x)^{n}=0$ for all $x \in \mathbb{C}^{n}$. By the reduction of degree theorem (Yagzhev [12], Bass, Connell and Wright [1], and Drużkowski [5]) it is known that the Jacobian conjecture is true if and only if it holds for the Yagzhev case.

Let us see how the Yagzhev form might simplify the iterated differentials of the identity $f\left(f^{-1}(x)\right) \equiv x$. First of all write $g(x)=-G(x, x, x)$ for a trilinear symmetric $G: \mathbb{C}^{n} \times \mathbb{C}^{n} \times \mathbb{C}^{n} \rightarrow \mathbb{C}^{n}$. The identity becomes

$$
f^{-1}(x)-G\left(f^{-1}(x), f^{-1}(x), f^{-1}(x)\right) \equiv x .
$$

We use the notation $\partial^{m} h(x)\left[u_{1}, \ldots, u_{m}\right]$ for the $m$ th differential of $h$ at $x$ applied to the $m$-tuple of vectors $\left(u_{1}, \ldots, u_{m}\right)$. The first three successive differentials of the identity are

$$
\begin{aligned}
\partial^{1} f^{-1}(x)\left[u_{1}\right] & -3 G\left(\partial^{1} f^{-1}(x)\left[u_{1}\right], f^{-1}(x), f^{-1}(x)\right) \equiv u_{1}, \\
\partial^{2} f^{-1}(x)\left[u_{1}, u_{2}\right] & -3 G\left(\partial^{2} f^{-1}(x)\left[u_{1}, u_{2}\right], f^{-1}(x), f^{-1}(x)\right) \\
& -6 G\left(\partial^{1} f^{-1}(x)\left[u_{1}\right], \partial^{1} f^{-1}(x)\left[u_{2}\right], f^{-1}(x)\right) \equiv 0, \\
\partial^{3} f^{-1}(x)\left[u_{1}, u_{2}, u_{3}\right] & -3 G\left(\partial^{3} f^{-1}(x)\left[u_{1}, u_{2}, u_{3}\right], f^{-1}(x), f^{-1}(x)\right) \\
& -6 G\left(\partial^{2} f^{-1}(x)\left[u_{1}, u_{2}\right], \partial^{1} f^{-1}(x)\left[u_{3}\right], f^{-1}(x)\right) \\
& -6 G\left(\partial^{2} f^{-1}(x)\left[u_{1}, u_{3}\right], \partial^{1} f^{-1}(x)\left[u_{2}\right], f^{-1}(x)\right) \\
& -6 G\left(\partial^{1} f^{-1}(x)\left[u_{1}\right], \partial^{2} f^{-1}(x)\left[u_{2}, u_{3}\right], f^{-1}(x)\right) \\
& -6 G\left(\partial^{1} f^{-1}(x)\left[u_{1}\right], \partial^{1} f^{-1}(x)\left[u_{2}\right], \partial^{1} f^{-1}(x)\left[u_{3}\right]\right) \\
&
\end{aligned}
$$


If we bear in mind that $f(0)=0$ we see that the first of these identities gives $\partial f^{-1}(0)\left[u_{1}\right] \equiv u_{1}$, whilst the second gives $\partial^{2} f^{-1}(0)\left[u_{1}, u_{2}\right] \equiv 0$. The third one gives $\partial^{3} f^{-1}(0)=6 G$. If we go on this way we see that the highest order differential of $f^{-1}$ appears always twice, first by itself and then as the first argument of a $G$. But the other arguments of $G$ in that term are always $f^{-1}(x)$, which vanishes for $x=0$. Then the $m$ th differential of $f^{-1}$ at the origin can always be expressed in terms of sums of $G$ 's having lower-order differentials of $f^{-1}$ as arguments. The following explicit recursive formula has been proved by Drużkowski and Rusek [7], expressed in terms of the homogeneous terms $\Phi_{m}(u):=m !^{-1} \partial^{m} f^{-1}(0)[u, u, \ldots, u]$ of the Taylor expansion of $f^{-1}$ at the origin:

$$
\begin{aligned}
\Phi_{1} & =I, \\
\Phi_{2 m+1} & =\sum_{p+q+r=m-1} G\left(\Phi_{2 p+1}, \Phi_{2 q+1}, \Phi_{2 r+1}\right), \\
\Phi_{2 m} & =0 \quad \text { for } m \in \mathbb{N} .
\end{aligned}
$$

We then find that $\partial^{2 m+1} f^{-1}(0)$ is a linear combination of terms, each of which is a nested composition in which $G$ appears exactly $m$ times. For example, $\Phi_{7}(u)$ is a linear combination of the two nestings

$$
G(G(G(u, u, u) u, u), u, u) \quad \text { and } \quad G(G(u, u, u), G(u, u, u), u),
$$

$\Phi_{9}(u)$ is a linear combination of the four nestings

$$
\begin{aligned}
& G(G(G(G(u, u, u), u, u) u, u), u, u), G(G(G(u, u, u), G(u, u, u), u), u, u), \\
& G(G(G(u, u, u), u, u), G(u, u, u), u), G(G(u, u, u), G(u, u, u), G(u, u, u)),
\end{aligned}
$$

and so on.

Here came the encouraging observation: the nilpotency condition $g^{\prime}(u)^{n}$ $\equiv 0$ means that when we nest the function $G(\cdot, u, u)$ at least $n$ times we get zero. Thus we have got rid of infinitely many of the nestings that make up the Taylor expansion of $f^{-1}$. What about the other nestings, the ones where at least two $G$ 's show up at the same level? One was drawn to wonder whether there may be some reason for all of them but finitely many to vanish too.

The point of this paper is to show that such hunch is wrong: there are polynomial automorphisms of Yagzhev form for which there are nonvanishing nestings beyond any arbitrary level.

The ground for our claim is a by-product of the following question related to the Jacobian conjecture, that was introduced in [4]. Given a real number $\lambda>1$ and a polynomial mapping $f$ not necessarily of Yagzhev type but with $f(0)=0, f^{\prime}(0)=I$ and $\operatorname{det} f^{\prime}(x)=1$ everywhere, it was proved that there exists a global analytic function $k_{\lambda}: \mathbb{C}^{n} \rightarrow \mathbb{C}^{n}$ such that $k_{\lambda}(0)=0$, 
$k_{\lambda}^{\prime}(0)=I$ and

$$
\lambda f\left(k_{\lambda}(y)\right)=k_{\lambda}(\lambda y) \quad \text { for all } y \in \mathbb{C}^{n} .
$$

This is a consequence of the Poincaré-Siegel theorem or of a sharper ad-hoc argument, as was done in [4]. Such a $k_{\lambda}$ "conjugates" $\lambda f$ to its linear part in a neighbourhood of the origin or even on the whole $\mathbb{C}^{n}$ if $k_{\lambda}$ is one-to-one and onto. The original motivation of considering $k_{\lambda}$ was that whenever it is one-to-one and onto then $f$ is too.

What concerns us here is the fact that, when $f$ is of Yagzhev form, the successive differentials of $k_{\lambda}$ are obtained in pretty much the same way as the differentials of $f^{-1}$, because the relation (7) can be written as

$$
\frac{\lambda k_{\lambda}(y)-k_{\lambda}(\lambda y)}{\lambda}-G\left(k_{\lambda}(y), k_{\lambda}(y), k_{\lambda}(y)\right) \equiv 0 .
$$

To perform the same calculations as for $f^{-1}$ we just have to keep in mind that $k_{\lambda}(0)=0$ and that $\lambda \neq 1$, so that at every step we can isolate the highest order differential as a function of the lower-order differentials.

LEMMA. The following recursion holds for the homogeneous terms $\Psi_{m}(u):=m !^{-1} \partial^{m} k_{\lambda}(0)[u, u, \ldots, u]$ of the Taylor expansion of $k_{\lambda}$ :

$$
\begin{aligned}
\Psi_{1}: & =I, \\
\Psi_{2 m+1} & =\frac{1}{1-\lambda^{2 m}} \sum_{p+q+r=m-1} G\left(\Psi_{2 p+1}, \Psi_{2 q+1}, \Psi_{2 r+1}\right), \\
\Psi_{2 m} & =0 \quad \text { for } m \in \mathbb{N} .
\end{aligned}
$$

The function $\Psi_{2 m+1}$ is then a linear combination of the very same nested compositions that make up $\Phi_{2 m+1}$.

Next, Arno van den Essen [8] has given the following example of a bijective polynomial mapping of $\mathbb{C}^{4}$ :

$$
\begin{aligned}
& f(x):=\left(x_{1}+p(x) x_{4}, x_{2}-p(x) x_{3}, x_{3}+x_{4}^{3}, x_{4}\right), \quad \text { where } \\
& p(x):=x_{3} x_{1}+x_{4} x_{2},
\end{aligned}
$$

for which the mapping $k_{\lambda}$ is not a polynomial. Later in [9] we proved that this $k_{\lambda}$ is one-to-one and onto, a result that Bo Deng has reobtained with a different approach in [3]. In [9] we also explicitly calculated the power series of $k_{\lambda}$.

Anyway, since this particular $k_{\lambda}$ is not a polynomial, there are integers $m$ beyond any arbitrary bound for which $\Psi_{2 m+1}$ does not vanish identically. Hence for those $m$ there must be some $m$-fold nestings of $G$ that do not vanish either, because a linear combination of them is nonzero.

We can now put to rest the hope to explain the polynomiality of $f^{-1}$ by proving that all nestings with sufficiently many $G$ 's inside were identically 
zero. This observation may possibly comfort the people who are skeptical about the Jacobian Conjecture itself.

We conclude with a formal statement of our result, preceded by a precise definition of the set $\operatorname{Nest}_{\Delta}(G, n)$ of "nested compositions" of $G$.

Definition. Given a set $V$, functions $T: V \times V \times V \rightarrow V$ and $M: V^{2 n+1} \rightarrow V$ and an index $i \in\{1, \ldots, 2 n+1\}$ we define the function $\widehat{M}_{i}: V^{2 n+3} \rightarrow V$ as

$$
\begin{aligned}
\widehat{M}_{i}\left(x_{1}, \ldots, x_{2 n+3}\right) \\
\quad:=M\left(x_{1}, \ldots, x_{i-1}, T\left(x_{i}, x_{2 n+2}, x_{2 n+3}\right), x_{i+1}, \ldots, x_{2 n+1}\right) .
\end{aligned}
$$

Then we define by induction

$$
\begin{aligned}
\operatorname{Nest}(T, 1) & :=\{T\}, \\
\operatorname{Nest}(T, n+1) & :=\left\{\widehat{M}_{i}: M \in \operatorname{Nest}(T, n), i=1, \ldots, 2 n+1\right\} .
\end{aligned}
$$

Then Nest $_{\Delta}(T, n)$ will be the set of functions $V \rightarrow V$ of the form $x \mapsto$ $M(x, \ldots, x)$, where $M \in \operatorname{Nest}(T, n)$.

Theorem. Let $G: \mathbb{C}^{n} \times \mathbb{C}^{n} \times \mathbb{C}^{n} \rightarrow \mathbb{C}^{n}$ be the symmetric trilinear form associated with the homogeneous third-degree term of the mapping $f$ of (10). Then there are infinitely many $n$ for which $\operatorname{Nest}_{\Delta}(G, n) \neq\{0\}$.

\section{References}

[1] H. Bass, E. Connell and D. Wright, The Jacobian conjecture: reduction of degree and formal expansion of the inverse, Bull. Amer. Math. Soc. 7 (1982), 287-330.

[2] A. Białynicki-Birula and M. Rosenlicht, Injective morphisms of real algebraic varieties, Proc. Amer. Math. Soc. 13 (1962), 200-203.

[3] B. Deng, Automorphic conjugation, global attractor, and the Jacobian conjecture, University of Nebraska-Lincoln, 1995.

[4] B. Deng, G. H. Meisters and G. Zampieri, Conjugation for polynomial mappings, Z. Angew. Math. Phys. 46 (1995), 872-882.

[5] L. M. Drużkowski, An effective approach to Keller's Jacobian conjecture, Math. Ann. 264 (1983), 303-313.

[6] - The Jacobian conjecture, Institute of Mathematics, Polish Academy of Sciences, preprint 492 (1991).

[7] L. M. Drużkowski and K. Rusek, The formal inverse and the Jacobian conjecture, Ann. Polon. Math. 46 (1985), 85-90.

[8] A. van den Essen (ed.), Automorphisms of Affine Spaces, Proc. of the Curaçao Conference, Kluwer Acad. Publ., 1985.

[9] G. Gorni and G. Zampieri, On the existence of global analytic conjugations for polynomial mappings of Yagzhev type, J. Math. Anal. Appl., to appear.

[10] O. H. Keller, Ganze Cremona Transformationen, Monatsh. Math. Phys. 47 (1939), 299-306.

[11] W. Rudin, Injective polynomial maps are automorphisms, Amer. Math. Monthly 102 (1995), 540-543. 
[12] A. V. Yagzhev, Keller's problem, Siberian Math. J. 21 (1980), 747-754.

Università di Udine

Dipartimento di Matematica e Informatica

Via delle Scienze 208

33100 Udine, Italy

E-mail: gorni@dimi.uniud.it
Università di Messina

Dipartimento di Matematica

Salita Sperone 31

98166 Sant'Agata, Messina, Italy

E-mail: Gaetano@pdmat1.unipd.it

Reçu par la Rédaction le 23.10.1995

Révisé le 7.2.1996 